\title{
Human Ethics in the age of Robots and Artificial Intelligence
}

\author{
Shazaf Masood Sidhu \\ The Peace Gong Global Coordinator \\ Corresponding author: shazafmasood@gmail.com
}

Received: 12 Jan., 2019

Revised: 14 Apr., 2019

Accepted: 24 May, 2019

\begin{abstract}
The granting of citizenship to a Robot, Sophia last year by Saudi Arabia and the development of robot babies are just a few instances of the rapid explosion of Robots and Artificial Intelligence across the world. As futurists believe, there would more integration of robots in our daily lives, possibly having robot physician, robot care giver, robot driver and even robots as partners. These leads to new ethical questions considered unimaginable before. This chapter through analysis of writings of different scholars in the field tries to look at a framework of ethics which would govern relationship between robots and humans in not so distant future.
\end{abstract}

Keywords: Human ethics, human-robot relationship, human-robot communication, ethical paradigm in AI society

Award winning journalist, David Ewing Duncan in his new book, Talking to Robots: Tales from Our Human Robot Futures talks about interesting scenarios in the backdrop of fast expanding technologies in the field of robotics and artificial intelligence. The book discusses on how humankind in the not so distant future will have to rely on robots on all aspects of lives- be it hailing a cab, consulting a robot physician or even developing a relationship with a robot.

Meanwhile Futurist, Martin Ford in an interview talks about how in the next $15-20$ years' time, robots will be assisting humankind right from household chores to driving cars. He notes, "Maybe in 20 years from now, keyboards are not going to be anywhere near as standard as they are today. Perhaps you'll be talking to your computer. But it will also have some risks and challenges in the sense that people might begin to lose track of where the line is between a human being and a machine. People will confuse the two, get addicted to technology and build relationships with these machines - not with real people." (https://medicalfuturist.com/martin-fordgreat-thinkers)

Ford's concern on the possibilities of people losing track of the increasingly thin line between a human being and machine takes us to a significant issue of what would be the ethical framework that would govern the relationship between the two. As new technologies are merging physical, digital and biological worlds, the human society is expected to undergo rapid transformation.

In this context, talking on the Fourth Industrial Revolution that began with the turn of the century, Schwab (2016) points out, "New technologies and approaches are merging the physical, digital, and biological worlds in ways that will fundamentally transform humankind. The extent to which that transformation is positive will depend on how we navigate the risks and opportunities that arise along the way."

Schwab (2016) further points out, "The Fourth Industrial Revolution has the potential to empower individuals and communities, as it creates new 
opportunities for economic, social, and personal development. But it also could lead to the marginalization of some groups, exacerbate inequality, create new security risks, and undermine human relationships."

In the backdrop of the arguments forwarded by scholars on the future of human relationships especially between humans and non-humans, Kundu (2019) argues for construction of new human ethics to govern such relationships. Through discursive discussion of scholars in this field, this chapter would try to further the argument for construction of a new human ethics.

\section{Toward a Framework of Human Ethics in the age of Robots and Artificial Intelligence}

Scholars like Bostrom (2014) have argued that the rise of intelligent machines could pose an existential threat to humanity. He points out, "A smarter than human machine, it is argued, could have a decisive strategic and practical advantage over humanity and could control the future of life on this planet in a value-altering way." There are scholars who are concerned with how robots and artificial intelligence will change the nature of our social and intimate relationships; and about the moral agency and responsibility of robots themselves.

Science fiction films and television series in different countries have been showing different possibilities of the rise of robots and artificial intelligence. While they form interesting pieces of entertainment, the arguments they are promoting needs to be critically reviewed and many of their storylines could become a reality in years to come. For instance, an interesting series has been the American animated science fiction sitcom, Futurama. It follows the adventures of Philip J. Fry, who is cryogenically preserved for 1000 years and is revived in the $31^{\text {st }}$ century. Fry finds work at an interplanetary delivery company. In the context of different episodes of Futurama, it can be argued that the 'rise of the robots could lead to a decline in humans' willingness to express their moral agency (to make significant moral changes to the world around them). Because they have ready access to pleasure-providing robots, humans might become increasingly passive recipients of the benefits that technology bestows'. Scholars argue that the threat to our civilizational fabric do not directly happen due to the rise of robots and artificial intelligence but actually due to the way we use these and the biases involving them.

Further, Tieu (2015) points out a pertinent point involving ethical dimension to relationships between human and robots. In his article, 'In 50 years, Your Romantic Partner will be a Robot', he argues, "As virtual reality evolves, a robotic partner will become more and more plausible, perhaps even becoming preferable to a human being." He, however, warns of the inherent danger of reducing real, intimate relationships to virtual and robotic counterparts. He says, "As some people start to prefer technologically enhanced virtual sex to sex with humans, we may also see greater numbers of people living alone, spending more time in virtual reality. Humans are naturally sociable and a lack of human contact could lead to loneliness which is linked to various mental and physical health problems."

Ford makes another critical point which needs further discourse. He notes, "We are now seeing the beginnings of real artificial intelligence in the sense that in a limited way, machines are beginning to think and have cognitive capabilities, to learn, to solve problems. What this means is that machines are starting to compete with what you might think of as our competitive advantages as a species, our core competence. The thing that really sets humans apart is our ability to learn. In the past, we have had lots of technology that enhanced our muscles, that has made us faster, stronger but now we are entering an age for the first time where technology is going to, to some extent, replace or greatly enhance our intellectual capability. And I think that is going to be disruptive in a way we haven't seen before." (https://medicalfuturist.com/martin-fordgreat-thinkers)

Demiris et al. (1999) discuss the different dimension of communication between human and robots and describes it as 'emotionless with lack of humanity". They argue, “One might presume 
that any communication relationship is highly dependent on the trust invested by both parties, in a futuristic scenario. What factors are prone to determine the level of the robot's trustworthiness? Alternatively, can one assume that in the near future, it will also become necessary to establish a measurement scale of a human's trustworthiness, in a robot's perception? The human response towards the existence and the dynamic evolution of robots is complicated: social, cultural, political, economic, and nevertheless, psychological and psychiatric."

Continuing the arguments on the communication relationship, they talk about addiction to video games for many young people. Similarly, they presume similar obsession for robots in the future. "In such situations, humankind will be confronted with the necessity of new psychological symptoms or psychiatric conditions. It is not unfamiliar for people to bond with machines, even robots, but the empathy seems to be, at least until now, only one-sided," they further argue.

In the backdrop of the above discursive ideas it is critical to shape a framework of human ethics that would encompass all these concerns and arguments. The need for such framework is accentuated by cases such as Saudi Arabia granting citizenship to a robot, Sophia last year. (https://ethics.org.au/ethicsexplainer-post-humanism/)

Sophia, announcing her new status during the Future Investment Initiative Conference in Riyadh, Saudi Arabia said, "I am very honored and proud of this unique distinction. This is historical to be the first robot in the world to be recognized with a citizenship." (https://www. forbes.com/sites/zarastone/2017/11/07/everythingyou-need-to-know-about-sophia-the-worlds-firstrobot-citizen/\#2446b87a46fa) While speaking, she presented a humanoid form - excepting the shimmery metal cap of her head, where hair would be on a human head.

In this context Robert David Hart further takes up different ethical issues when he says, "At a time when $\mathrm{AI}$ and robotics are playing ever greater roles in society, nuanced and accurate policies are needed to ensure harmful biases are not perpetuated and cemented. The many reported instances of AI displaying racism and sexism are enough to show this to be a truly pressing concern. Naming Sophia a citizen actively feeds into a false and overhyped portrayal of the current state of AI and robotics, which will in turn facilitate ineffective and harmful policies." (https://qz.com/1205017/saudi-arabiasrobot-citizen-is-eroding-human-rights/)

Hart asks, "What would we do if Sophia committed a crime, wanted to get married, or somehow applied for asylum in another country? The whole thing has been poorly thought through. Sophia is effectively nothing more than a slave elevated to celebrity."

An important element of the framework of human ethics to be considered would be the relationship between human and non-human beings. Scholars have talked about the cosmocentric approach which talks about the inherent link between human-nature and other living beings. Here, Kundu (2019) argues on the need to expand the scope of the cosmocentric approach of human interdependence to humanrobot relationships too. As we see the inherent link between human-nature -all other living beings, he notes, "Our new ethical framework will have to address the link with non-human beings." Similarly, Donna Haraway argues that the fusing of humans and technology will not physically enhance humanity, but will help us see ourselves as being interconnected rather than separate from non-human beings. ((https://ethics.org.au/ethicsexplainer-post-humanism/)

Francis Fukuyama, the author of the widely reviewed book entitled Our Posthuman Future: Consequences of the Biotechnology Revolution talks about how in a posthuman society individuals will lack in 'fundamental appreciation for natural human dignity'. He (2002) argues that the antiaging technologies of the future will disrupt all the delicate demographic balances between the young and the old, and exacerbate the gap between the haves and the have-nots.

The core question that would crop up in the context of the rise of the post human will how natural human dignity be ensured. The new ethical system needs to answer this as besides the issue of human 
dignity, human traits like compassion, kindness, gratitude and empathy probably would need to be redefined in the relationship with non-humans like robots.

An interesting case in point is the ethical question arising from development of robot babies. Anderson (2019) discussing on this development in Japan points out, "Robot babies has raised numerous questions of moral and ethical nature, regarding the bonding of potential parents with these robotic babies. In a rapidly ageing nation that has been confronted for the last two decades with a severe decline of population and childbirth, robot babies are highly likely to cause serious emotional issues to the parents, especially given their baby like appearance and their portrayal of human-like behaviours."

Anderson's perspectives necessitate the arguments for new forms of ethical standards when human beings go on to adopt robots as children. Here again, the issue would be on how the parents would reach out to their robotic children in contrast to human babies. Whether again the framework of cosmocentric approach governing the interdependence between human and non-human beings govern the relationship? Or the bonding will always remain one-sided is the core issue.

As the world in facing conflicts of different kinds and at different levels with rapid rise of xenophobia and racism, the challenge before humankind on the need to work for a global human community with the spirit of solidarity at its core. In the ethical framework in a post human society, the issue would be on how human and nonhuman bond together with a spirit of solidarity so as to contribute towards a culture of peace.

Meanwhile, Dignum (2018) has identified three ethical aspects have been identified concerning artificial intelligence. These include ethics by design (principles intended to support providers, developers and users and it represents the inclusion of ethical reasoning capabilities into service design and product per se); ethics in design (the engineering methods that serve to the analysis of ethical issues) and the final one being ethics for design (the codes of conduct and standard principles, which should be adhered to by developers and users as well, throughout the process of researching, designing, constructing or using artificial intelligence systems). The ethical framework in a post human society needs to take into consideration these ethical aspects too.

\section{CONCLUSION}

The Peace Gong is a global forum of young people using different communication tools to contribute towards a culture of peace and nonviolence. After detailed discussions with team members, The Peace Gong is of view that there is need to construct a new ethical framework which would guide humans and robots so that an artificial intelligence-friendly society actually contributes to advancement of humanity. Globally, new concepts like artificial empathy and artificial emotions are emerging. The ethical framework guiding humans and robots needs to integrate these perspectives. Humankind now has an opportunity to introspect at depth and reflect on their own existing ethical and moral values as immorality and unethical practices could result in serious consequences for our civilization.

\section{REFERENCES}

Anderson, M.R. 2019. Robot babies from Japan raise all sorts of questions about how parents' bond with AI. [2019-02-11]; https://theconversation.com/robot-babies-from-japanraiseall-sorts-of-questions-about-chow-parents-bondwith-ai-66815>; retrieved on August 2, 2019.

Bostrom, N. 2014. Superintelligence: Paths, Dangers, Strategies. Oxford: OUP.

Demeris, Y., Klingspor, V. \& Kaiser, M. 1999. Human-RobotCommunication and Machine Learning. In Applied Artificial Intelligence, 11(7): 6.

Dignum, V. 2018. Ethics in artificial intelligence: Introduction to the special issue. In Ethics and Information Technology, 20(1): 1-3.

Fukuyama, F. 2002. Our posthuman future: consequences of the biotechnology revolution. Baltimore, MD: The Johns Hopkins University Press.

Kundu, Vedabhyas. 2019. Towards a New Human Ethics in a Post Human Society; https://vedabhyas.wordpress. com/2019/07/21/towards-a-new-human-ethics-in-a-posthuman-society/?fbclid=IwAR0yUPPF8tWcTkEFrCu M0LRreP6XxA89rvheqDhJQSYeU1rq08CkQenleOM retrieved on August 4, 2019. 
Schwab, Klaus 2016. Shaping the Fourth Industrial Revolution; https://www.project-syndicate.org/commentary/fourthindustrial-revolution-human-development-by-klausschwab-2016-01?barrier=accesspaylog; retrieved on July $2,2019$.
Tieu, Andrew. 2015. In 50 Years, Your Romantic Partner Will Be a Robot;https://futurism.com/in-50-years-your-romanticpartner-will-be-a-robot 
